\title{
ON SYMMETRIC MATRICES WHOSE EIGENVALUES SATISFY LINEAR INEQUALITIES ${ }^{1}$
}

FRITZ JOHN

The nonnegative symmetric matrices $\left(a_{i k}\right)$ of order $n$ are those for which

$$
\sum_{i, k=1}^{n} a_{i k} \xi_{i} \xi_{k} \geqq 0 \quad \text { for all } \xi,
$$

at the same time these are also the symmetric matrices whose eigenvalues $\lambda_{i}$ satisfy

$$
\lambda_{1} \geqq 0, \cdots, \lambda_{n} \geqq 0 .
$$

Moreover every such matrix can be represented as a sum of $n$ matrices of the same type that have rank $\leqq 1$ (corresponding to the representation of definite forms as sums of squares of linear forms).

We have here an instance of a system of linear inequalities (1b) for the eigenvalues of a matrix that is equivalent to a system of linear inequalities (1a) for the elements of the matrix. The present note shows that generally systems of linear inequalities for the eigenvalues of a symmetric matrix (satisfied irrespective of the arrangement of the eigenvalues) are equivalent to suitable systems of linear inequalities for the matrix elements. ${ }^{2}$ Instead of solutions of systems of linear inequalities we shall talk of the convex sets formed by such solutions.

The general real $n$th order matrix $a=\left(a_{i k}\right)$ will be represented by a point in $E_{m}$ where $m=n^{2}$. Let $\Sigma$ be the set of real symmetric matrices of order $n$ and $\Omega$ that of real orthogonal matrices. Each $a \in \Sigma$ gives rise to an unordered set of $n$ real eigenvalues that can be represented by points in $E_{n}$. For given $a \in \Sigma$ we denote by $\Lambda_{a}$ the set of all points $\lambda=\left(\lambda_{1}, \cdots, \lambda_{n}\right)$ in $E_{n}$ whose coordinates $\lambda_{i}$ are the eigenvalues of $a$ in some order. ${ }^{3}$

Conversely we denote for a given $\lambda=\left(\lambda_{1}, \cdots, \lambda_{n}\right)$ in $E_{n}$ by $A_{\lambda}$ the set of all symmetric matrices $a$ whose eigenvalues suitably arranged

Received by the editors October 17, 1965.

1 This research was supported by the Office of Naval Research under Contract No. Nonr-285 (46). Reproduction in whole or in part is permitted for any purpose of the U.S. Government.

2 The restriction to symmetric matrices is essential. For example positiveness of the eigenvalues of a general matrix is not expressible by linear inequalities on the elements.

The set $\bigwedge_{a}$ has at most $n$ ! elements. 
are $\lambda_{1}, \cdots, \lambda_{n}$. We associate with a point $\lambda=\left(\lambda_{1}, \cdots, \lambda_{n}\right)$ in $E_{n}$ the diagonal matrix with diagonal elements $\lambda_{1}, \cdots, \lambda_{n}$ which we shall denote by $[\lambda]$. The relations

$$
\lambda \in \Lambda_{a} \text { or } a \in A_{\lambda}
$$

are then equivalent to the existence of an orthogonal matrix $c$ such that ${ }^{4}$

$$
a=c[\lambda] c^{T} .
$$

The correspondence between $\lambda$ and $a$ implied by $(2 \mathrm{a}, \mathrm{b})$ leads naturally to two kinds of mappings of sets in $E_{n}$ onto subsets of $\Sigma$ in $E_{m}$. Given a set $\sigma$ of points in $E_{n}$ we define the sets ${ }^{5}$

$$
\begin{aligned}
& C(\sigma)=\left\{a \mid a \in \Sigma, \wedge_{a} \in \sigma\right\}, \\
& D(\sigma)=\left\{a \mid a \in \Sigma, a \in A_{\lambda} \text { for some } \lambda \in \sigma\right\} .
\end{aligned}
$$

THEOREM. If $\sigma$ is a closed convex set in $E_{n}$ then $C(\sigma)$ is closed and convex. If the closed convex set $\sigma$ is invariant under all permutations of coordinate axes and is the convex hull of a set $\tau$ then $C(\sigma)$ is the convex hull of $D(\tau)$. If here $\tau$ is a cone with vertex at the origin then $C(\sigma)$ is the set of all matrices that are representable as sum of $n$ matrices in $D(\tau)$.

(The representation of nonnegative quadratic forms as sums of $n$ squares is a special case. Here $\sigma$ is the set in $E_{n}$ described by (1b). We can choose for $\tau$ the subset where all but one of the $\lambda_{i}$ vanish. Then $D(\tau)$ consists of the nonnegative matrices of rank $\leqq 1$.)

Proof of THE Theorem. A closed convex set $\sigma$ can be represented as intersection of closed half-spaces. There exists then a system of linear inequalities

$$
\mu_{1} \lambda_{1}+\cdots+\mu_{n} \lambda_{n} \geqq p
$$

with coefficients $\left(\mu_{1}, \cdots, \mu_{n}, p\right)=(\mu, p)$ forming a set $M$ in $E_{n+1}$ that completely characterises the points $\lambda=\left(\lambda_{1}, \cdots, \lambda_{n}\right)$ of $\sigma$. For a given $(\mu, p)$ the points $\lambda$ satisfying (3) form a closed half-space $H_{\mu, p}$. We have

4 The superscript $T$ denotes transposition.

- $C(\sigma)$ is the set of symmetric matrices whose eigenvalues in all possible arrangements represent points of $\sigma$, while $D(\sigma)$ is the set of symmetric matrices whose eigenvalues when suitably arranged represent points in $\sigma$. We have $C(\sigma)=D(\sigma)$ when the set $\sigma$ is invariant under all permutations of coordinates. 


$$
\sigma=\bigcap_{(\mu, p) \in M} H_{\mu, p}, \quad C(\sigma)=\bigcap_{(\mu, p) \in M} C\left(H_{\mu, p}\right) .
$$

To show closedness and convexity of $C(\sigma)$ it is sufficient to show that the set $C\left(H_{\mu, p}\right)$ is closed and convex for each $\mu, p$. It suffices to prove that $C\left(H_{\mu, p}\right)$ is identical with the set of matrices $a \in \Sigma$ satisfying the system of linear inequalities

$$
\operatorname{trace}(a b) \geqq p \quad \text { for all } b \in A_{\mu} .
$$

Let indeed $a$ be a symmetric matrix satisfying (4) and let $\lambda \in \Lambda_{a}$. Then $a=c[\lambda] c^{T}$ for some $c \in \Omega$. We have

$$
b=c[\mu] c^{T} \in A_{\mu}
$$

and hence

$$
\operatorname{trace}(a b)=\operatorname{trace}([\lambda][\mu])=\sum_{i=1}^{n} \lambda_{i} \mu_{i} \geqq p .
$$

Thus $\lambda \in H_{\mu, p}$ whenever $\lambda \in \Lambda_{a}$ which proves that $a \in C\left(H_{\mu, p}\right)$.

Conversely assume that

$$
a \in C\left(H_{\mu, p}\right) .
$$

Let $\lambda \in \bigwedge_{a}$ and $b \in A_{\mu}$. Then

$$
a=c[\lambda] c^{T} \quad \text { and } \quad b=d[\mu] d^{T} \quad \text { where } \quad c, d \in \Omega .
$$

Denote by $e=\left(e_{i k}\right)$ the orthogonal matrix $e=c^{T} d$ and by $f=\left(f_{i k}\right)$ the matrix with elements $f_{\imath k}=e_{i k}^{2}$. Then

$$
\begin{aligned}
\operatorname{trace}(a b) & =\operatorname{trace}\left(c[\lambda] c^{T} d[\mu] d^{T}\right) \\
& =\operatorname{trace}\left([\lambda] e[\mu] e^{T}\right)=\sum_{i, k} f_{i k} \lambda_{i} \mu_{k} .
\end{aligned}
$$

Since $e$ is orthogonal the matrix $f$ is doubly-stochastic. ${ }^{6}$ By Birkhoff's theorem (see [1, p. 97]) the set of doubly-stochastic matrices forms a convex polyhedron in $E_{m}$ with the permutation matrices as vertices. Hence there exist nonnegative numbers $\epsilon_{1}, \cdots, \epsilon_{N}$ of sum 1 such that

$$
\sum_{j=1}^{n} f_{i k} \lambda_{i}=\sum_{j=1}^{N} \epsilon_{j} \lambda_{k}^{(j)},
$$

where for each $j$ the numbers $\lambda_{1}^{(j)}, \lambda_{2}^{(j)}, \cdots, \lambda_{n}^{(j)}$ form a permutation

${ }^{6}$ That is the elements of $f$ are nonnegative and those in any row or column add up to 1 . 
of the values $\lambda_{1}, \cdots, \lambda_{n}$. Since $\lambda^{(j)} \in \Lambda_{a}$ it follows from (5) that

$$
\operatorname{trace}(a b)=\sum_{j=1}^{N} \epsilon_{j} \sum_{k=1}^{n} \mu_{k} \lambda_{k}^{(j)} \geqq \sum_{j=1}^{N} p \epsilon_{j}=p .
$$

Thus a matrix $a \in C\left(H_{\mu, p}\right)$ satisfies (4). We have proved that $C(\sigma)$ is closed and convex.

Assume now that the closed convex set $\sigma$ is the convex hull of a set $\tau$, and that $\sigma$ is invariant under all permutations of coordinates. Then $C(\sigma)=D(\sigma)$. Since $\tau \subset \sigma$ we have

$$
D(\tau) \subset D(\sigma)=C(\sigma) .
$$

It follows from the proved convexity of $C(\sigma)$ that the convex hull of $D(\tau)$ is contained in $C(\sigma)$. Conversely let $a \in C(\sigma)$. Then $a$ is of the form $a=c[\lambda] c^{T}$ with $\lambda \in \sigma$ and $c \in \Omega$. Since $\lambda$ is in the convex hull of $\tau$ we can find nonnegative numbers $\epsilon_{1}, \cdots, \epsilon_{N}$ of sum 1 and points $\lambda^{(1)}, \cdots, \lambda^{(N)}$ in $\tau$ such that

$$
\lambda=\sum_{j=1}^{N} \epsilon_{j} \lambda^{(j)}
$$

Then

$$
a=\sum_{j=1}^{N} \epsilon_{j}\left[\lambda^{(j)}\right] c^{T}
$$

Here the matrices $c\left[\lambda^{(j)}\right] c^{T}$ belong to $D(\tau)$, and consequently $a$ lies in the convex hull of $D(\tau)$. It follows that $C(\sigma)$ is the convex hull of $D(\tau)$.

In the special case where $\tau$ is a cone ${ }^{7}$ with vertex at the origin we can assume that $N$ has the value $n$, since by Carathéodory's theorem (see $\left[2\right.$, p. 35]) any point of the convex hull of a connected set in $E_{n}$ is the centroid of $n$ nonnegative masses located in (not necessarily distinct) points of the set. We can write (6) in the form

$$
a=\sum_{j=1}^{n} c\left[\epsilon_{j} \lambda^{(j)}\right] c^{r}
$$

and have represented $a$ as sum of $n$ elements of $D(\tau)$, since with $\lambda^{(j)}$ also $\epsilon_{j} \lambda^{(j)}$ lies in $\tau$. Actually the sum of any number of matrices in $D(\tau)$ also lies in $C(\sigma)$ since it belongs to the convex hull of $D(\tau)$.

${ }^{7}$ The set $\tau$ is a cone with vertex at the origin if $\left(\lambda_{1}, \cdots, \lambda_{n}\right) \in \tau$ implies $\left(\gamma \lambda_{1}, \cdots\right.$, $\left.\gamma \lambda_{n}\right) \in \tau$ for any real $\gamma \geqq 0$. Cones are connected sets. 
ExAmple. Let $\sigma$ be the set of points $\lambda=\left(\lambda_{1}, \cdots, \lambda_{n}\right)$ described by the inequalities

$$
0 \leqq \lambda_{i} \leqq \frac{1}{2}\left(\lambda_{1}+\cdots+\lambda_{n}\right) \quad \text { for } i=1,2, \cdots, n .
$$

(For $n=3$ the system ( 7 ) is equivalent to the triangle inequalities

$$
\lambda_{1} \leqq \lambda_{2}+\lambda_{3}, \quad \lambda_{2} \leqq \lambda_{3}+\lambda_{1}, \quad \lambda_{3} \leqq \lambda_{1}+\lambda_{2} \text {.) }
$$

Obviously $\sigma$ is a closed convex set and invariant under permutations of the $\lambda_{t}$. Let $\tau$ be the set consisting of the points

$$
\lambda=(t, t, 0,0, \cdots, 0) \quad \text { with } t \geqq 0
$$

and all those obtained from (9) by permutation of coordinates. Clearly $\tau$ is a cone with vertex at the origin contained in $\sigma$. Hence the convex hull $\eta$ of $\tau$ also is contained in $\sigma$. Actually $\eta=\sigma$, for, as is easily seen, $\eta$ is closed. If $\eta \neq \sigma$ there would exist a point $\lambda=\left(\lambda_{1}, \cdots, \lambda_{n}\right)$ satisfying all inequalities (7) and also satisfying an additional inequality

$$
\mu_{1} \lambda_{1}+\cdots+\mu_{n} \lambda_{n} \geqq p
$$

which does not hold for any point in $\tau$. This implies that

$$
t\left(\mu_{i}+\mu_{k}\right)<p \quad \text { for } i \neq k \text { and all } t \geqq 0
$$

and consequently

$$
\mu_{i}+\mu_{k} \leqq 0<p \quad \text { for } i \neq k .
$$

If none of the quantities $\mu_{i}$ were positive relation (10) could not hold since $p>0$ and $\lambda_{i} \geqq 0$ for all $i$. If, say, $\mu_{1}>0$ it follows from (7), (11) that

$$
\begin{aligned}
\mu_{1}\left(\lambda_{1}-\lambda_{2}-\lambda_{3}-\cdots-\right. & \left.\lambda_{n}\right)+\left(\mu_{1}+\mu_{2}\right) \lambda_{2} \\
& +\left(\mu_{1}+\mu_{3}\right) \lambda_{3}+\cdots+\left(\mu_{1}+\mu_{n}\right) \lambda_{n} \leqq 0<p
\end{aligned}
$$

contrary to (10). Thus $\sigma$ is the convex hull of $\tau$.

From our theorem we find then that every matrix of $C(\sigma)$ is representable as sum of $n$ matrices in $D(\tau)$. A matrix $b$ belongs to $D(\tau)$ iff it is of the form $b=c[\lambda] c^{T}$ with $c \in \Omega$ and $\lambda$ given by (9). We can write $[\lambda]=-g^{2}$ where $g=\left(g_{i k}\right)$ is the skew matrix with elements

$$
g_{i k}=t^{1 / 2}\left(\delta_{i 2} \delta_{k 1}-\delta_{i 1} \delta_{k 2}\right) \text {. }
$$

Then $b=c[\lambda] c^{T}=-\left(c g c^{T}\right)^{2}$ where $c g c^{T}$ is again a skew matrix of rank $\leqq 2$. Hence

Every symmetric matrix of order $n$ whose eigenvalues $\lambda_{i}$ satisfy (7) can be represented as the negative of a sum of $n$ squares of real skew matrices of rank $\leqq 2$. 
Conversely we can show

The negative of any sum of squares of skew matrices is a symmetric matrix with eigenvalues satisfying (7). For any real skew matrix $g$ has eigenvalues that are either zero or conjugate pure imaginary in pairs. Then $-g^{2}$ is symmetric and has eigenvalues that are either 0 or positive and equal in pairs. The eigenvalues of $-g^{2}$ satisfy (7) so that $-g^{2} \in C(\sigma)$. Since $C(\sigma)$ is a convex cone with vertex at the origin any sum of matrices of the form $-g^{2}$ where $g$ is skew also lies in $C(\sigma)$.

Added in proof. An instance of equivalence of inequalities for matrix elements and for eigenvalues of the matrix is due to R. Hill [cf. C. Truesdell and R. A. Toupin, Correction to our paper "Static grounds for inequalities in finite strain of elastic materials," Arch. Rational Mech. Anal. 19 (1965), 407]. Hill finds that the third order symmetric matrices $a$ for which

$$
\text { trace }(g a g)=\operatorname{trace}\left(a g^{2}\right)<0
$$

for all skew symmetric $g \neq 0$ are exactly those whose eigenvalues $\lambda_{i}$ satisfy $\lambda_{i}+\lambda_{k}>0$ for $i \neq k$. This corresponds to our inequalities (3), (4) with $p=0$ and $\mu=(t, t, 0)$ where $t>0$.

\section{REFERENCES}

1. M. Marcus and H. Minc, $A$ survey of matrix theory and matrix inequalities, Allyn and Bacon, Boston, Mass., 1964.

2. H. G. Eggleston, Convexity, Cambridge Tracts in Mathematics and Mathematical Physics, No. 47, Cambridge Univ. Press, New York, 1958.

Courant Institute of Mathematical Sciences, New York University 